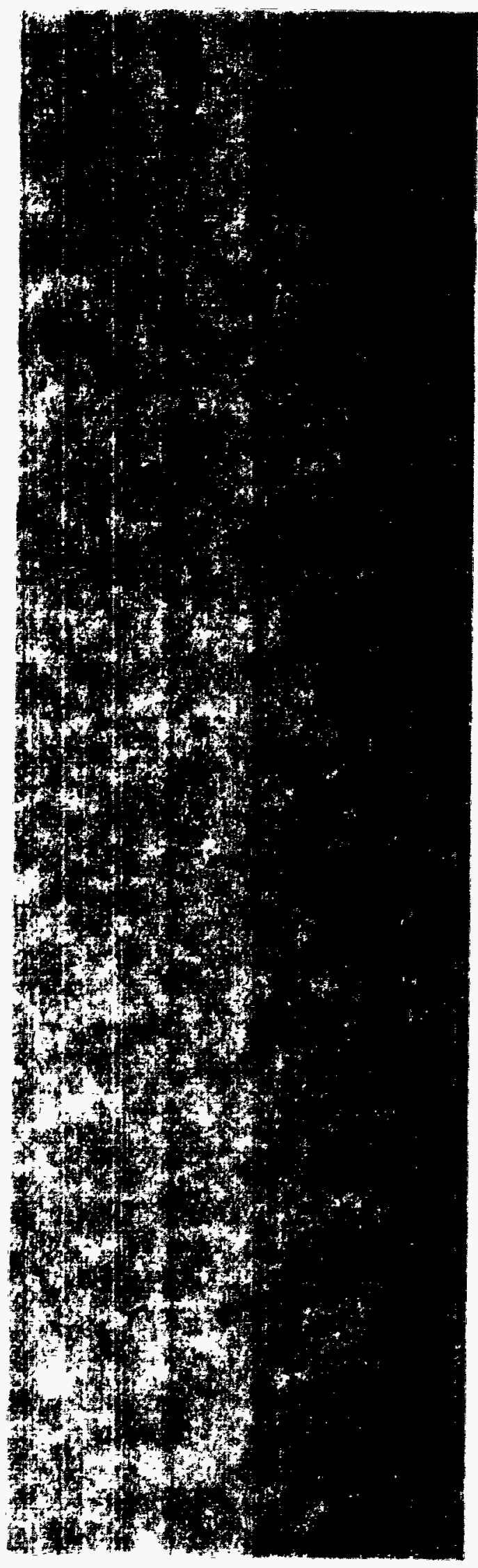

\title{
Propagation of Electromagnetic
}

Waves in a Structured Ionosphere

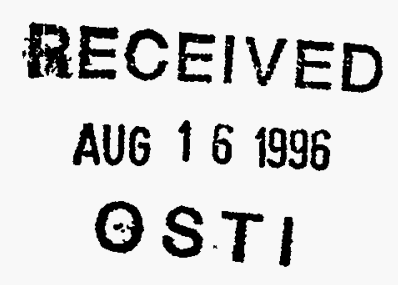

\section{Los Alamos \\ NATIONAL LABORATORY}

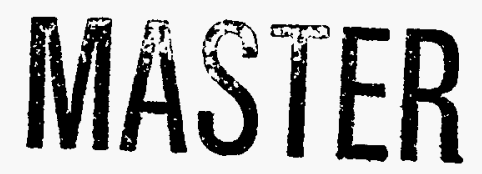

Los Alamos National Laboratory is operated by the University of Californin for the United States Department of Energy under contract W-7405-ENG-36. 
Edited by Brian Fishbine, CIC-1

An Affirmative Action/Equal Opportunity Employer

This report was prepared as an account of work sponsored by an agency of the United States Government. Neither The Regents of the University of Califomia, the United States Government nor any agency thereof, nor any of their employees, makes any warranty, express or implied, or assumes any legal liability or responsibility for the accuracy, conteleteness, or usefulness of any information, apparatus, product, or process disclosed, or represents that its use would not infringe privately owned rights. Reference herein to any specific commercial product, process, or service by trade name, trademark, manufacturer, or otherwise, does not necessarily constitute or imply its endorsement, recommendation, or favoring by The Regents of tine University of California, the United States Government, or any agency thereof. The views and opinions of authors expressed herein do not necessarily state or reflect those of The Regents of the University of California, the United States Government, or any agency thereof. The Los Alamos National Laboratory strongly supports academic freedom and a researcher's right to publish; therefore, the Laboratory as an institution does not endorse the viewpoint of a publication or guarantee its technical correctness. 
Propagation of Electromagnetic

Waves in a Structured Ionosphere

Tim Murphy 



\section{DISCLAIMER}

This report was prepared as an account of work sponsored by an agency of the United States Government. Neither the United States Government nor any agency thereof, nor any of their employees, makes any warranty, express or implied, or assumes any legal liability or responsibility for the accuracy, completeness, or usefulness of any information, apparatus, product, or process disclosed, or represents that its use would not infringe privately owned rights. Reference herein to any specific commercial product, process, or service by trade name, trademark, manufacturer, or otherwise does not necessarily constitute or imply its endorsement, recommendation, or favoring by the United States Government or any agency thereof. The views and opinions of authors expressed herein do not necessarily state or reflect those of the United States Government or any agency thereof. 


\section{DISCLAIMER}

Portions of this document may be illegible in electronic image products. Images are produced from the best available original document. 


\title{
Propagation of Electromagnetic Waves in a Structured Ionosphere
}

\author{
by \\ Tim Murphy
}

\begin{abstract}
The ionosphere is a birefringent medium which strongly affects the transmission of very high frequency (vhf) radio signals. These effects must be understood in detail if one wishes to look at the propagation of wide bandwidth coherent signals through the ionosphere. We develop a general perturbative solution of Maxwell's equations for vhf signals propagating in the ionosphere, subject only to mild restrictions on the ionospheric structure. This solution can be extended to give the propagating field to any desired degree of precision. The case of a laminar ionosphere with harmonic waves is developed in greater detail, and we show how to calculate the ray path in this case. This solution is used to elucidate the effects of refraction on the phase of the signal, and we calculate the spatial- and frequency-coherence functions. The electric field for a laminar ionosphere without waves is analyzed to clarify the physical origins of the terms modifying the signal phase. We then calculate the solution in this case for the Appleton-Hartree model of the ionospheric dielectric function and express the result as a series in inverse powers of frequency. We conclude by calculating the ray path for a model ionosphere using the Appleton-Hartree dielectric function and a parabolic layer for the electron density.
\end{abstract}

\section{Solution of the Wave Equation}

The ionosphere is a low-density plasma which occurs naturally in the upper atmosphere. For electromagnetic waves with frequencies above the plasma frequency, it behaves as a dielectric medium with a nonuniform dielectric function $\epsilon(\vec{x})$. In a uniform medium with a constant dielectric function, the effect of the dielectric is simply to slow the propagation of electromagnetic waves and to refract the wave, or change the direction of the wave propagation, at boundaries between regions of different dielectric constant. If the dielectric function is nonuniform, there is a continuous process of refraction. We need to understand this process in detail if we are to faithfully model the transmission of waves through the ionosphere. 
The propagation of electromagnetic waves is governed by Maxwell's equations for the electric field $\vec{E}$ and magnetic field $\vec{B}$ (Ref. 1). Although the ionosphere is ionized, the local charge density is approximately zero. There are large-scale current systems, but these are weak and vary slowly and do not play a role in the propagation of waves at the vhf frequencies of interest in this work, so we shall ignore these currents and assume that the local current density is zero. Thus we begin with Maxwell's equations for the fields $\vec{E}$ and $\vec{B}$ in a dielectric medium with zero net charge and current density, expressed in cgs units:

$$
\begin{aligned}
\vec{\nabla} \cdot(\epsilon \vec{E}) & =0, \\
\vec{\nabla} \cdot \vec{B} & =0, \\
\vec{\nabla} \times \vec{B}-\frac{1}{c} \frac{\partial(\epsilon \vec{E})}{\partial t} & =0, \\
\vec{\nabla} \times \vec{E}+\frac{1}{c} \frac{\partial \vec{B}}{\partial t} & =0 .
\end{aligned}
$$

Taking the curl of Eq. 4 and using Eq. 3 and standard vector identities, we have that

$$
\vec{\nabla} \times(\vec{\nabla} \times \vec{E})+\frac{1}{c} \frac{\partial(\vec{\nabla} \times \vec{B})}{\partial t}=\vec{\nabla}(\vec{\nabla} \cdot \vec{E})-\nabla^{2} \vec{E}+\frac{1}{c^{2}} \frac{\partial^{2}(\epsilon \vec{E})}{\partial t^{2}}=0 .
$$

We use Eq. 1 to replace the $\vec{\nabla} \cdot \vec{E}$ term and assume that the field is varying harmonically with angular frequency $\omega$ to arrive at the wave equation we wish to solve:

$$
\nabla^{2} \vec{E}(\vec{x})+\frac{\omega^{2}}{c^{2}} \epsilon(\vec{x}) \vec{E}(\vec{x})=-\vec{\nabla}\left[\frac{\vec{E}(\vec{x}) \cdot \vec{\nabla} \epsilon(\vec{x})}{\epsilon(\vec{x})}\right] .
$$

We are interested in solving this equation for a signal propagating from $z=-\infty$ to $z=\infty$, with or without motion in the $x$ and $y$ directions. In general there will be waves propagating in both the $+z$ and $-z$ directions, which we shall refer to as forward- and backward-going waves, respectively. We take the boundary condition to be that $\vec{E}$ is a purely forward-going wave at $z=\infty$ and that the forward-going component of $\vec{E}$ is of the form $\vec{E}=\vec{E}_{0} e^{i \vec{x} \cdot \vec{k}}$ at $z=-\infty$, with $|\vec{k}|=\omega / c, \vec{k} \cdot \vec{E}_{0}=0$, and $k_{z}>0$. The components of the wave vector in the $x$ and $y$ directions, $k_{x}$ and $k_{y}$, may or may not be zero. We assume that the dielectric function $\epsilon(\vec{x})$ goes to the constant value 1.0 as $z \rightarrow \pm \infty$.

As we shall demonstrate later, the backward-going component of $\vec{E}$ is very small if the frequency of the wave is well above the plasma frequency of the ionosphere. We shall therefore proceed as if there were no backward-going component at $z=-\infty$ and indicate how this component appears later in the calculation.

If the dielectric function of the ionosphere were 1.0 everywhere, the solution to the wave equation would be $\vec{E}=\vec{E}_{0} e^{i \vec{x} \cdot \vec{k}}$. The dielectric function is not uniform, but for frequencies well above the 
plasma frequency the deviations from the vacuum value are small. We therefore write the electric field in the form $\vec{E}=\vec{A}(\vec{x}) e^{i \vec{x} \cdot \vec{k}}$. Plugging this into Eq. 6, we find that

$$
\nabla^{2} \vec{A}+2 i(\vec{k} \cdot \vec{\nabla}) \vec{A}+k^{2}(\epsilon-1) \vec{A}=\frac{-i \vec{A}(\vec{k} \cdot \vec{\nabla} \epsilon)}{\epsilon}-(\vec{A} \cdot \vec{\nabla})\left(\frac{\vec{\nabla} \epsilon}{\epsilon}\right)-\left(\frac{\vec{\nabla} \epsilon \cdot \vec{\nabla}}{\epsilon}\right) \vec{A}-\frac{\vec{\nabla} \epsilon}{\epsilon} \times(\vec{\nabla} \times \vec{A})
$$

We will develop a perturbation series solution to this equation. It will make it easier to identify the order of various terms in the series if quantities are expressed in the dimensionless coordinates $\vec{x}^{\prime}=k \vec{x}$. In these coordinates the equation is

$$
\nabla^{\prime 2} \vec{A}+2 i\left(\hat{k} \cdot \vec{\nabla}^{\prime}\right) \vec{A}+(\epsilon-1) \vec{A}=\frac{-i \vec{A}\left(\hat{k} \cdot \vec{\nabla}^{\prime} \epsilon\right)}{\epsilon}-\left(\vec{A} \cdot \vec{\nabla}^{\prime}\right)\left(\frac{\vec{\nabla}^{\prime} \epsilon}{\epsilon}\right)-\left(\frac{\vec{\nabla}^{\prime} \epsilon \cdot \vec{\nabla}^{\prime}}{\epsilon}\right) \vec{A}-\frac{\vec{\nabla}^{\prime} \epsilon}{\epsilon} \times\left(\vec{\nabla}^{\prime} \times \vec{A}\right),
$$

where $\hat{k}$ is a unit vector parallel to $\vec{k}$. We shall consider the case where the dielectric function varies slowly on the scale of the wavelength of the electromagnetic wave. Formally, we have that

$$
R=\frac{|\vec{\nabla} \epsilon(\vec{x})|}{|\vec{k}|}=\left|\vec{\nabla}^{\prime} \epsilon(\vec{x})\right| \ll 1 .
$$

Because the right-hand side of the equation for $A$ is proportional to $R$, it is natural to look for a solution which is an expansion in powers of $\mathrm{R}$. We write $A=A_{0}+A_{1}+A_{2}+\ldots$, where $A_{n} \propto R^{n}$. Inserting this expansion into Eq. 8 and equating terms of the same order in $R$, we find that

$$
\begin{aligned}
\nabla^{\prime 2} \vec{A}_{0}+2 i\left(\hat{k} \cdot \vec{\nabla}^{\prime}\right) \vec{A}_{0}+(\epsilon-1) \vec{A}_{0}= & 0, \\
\nabla^{\prime 2} \vec{A}_{1}+2 i\left(\hat{k} \cdot \vec{\nabla}^{\prime}\right) \vec{A}_{1}+(\epsilon-1) \vec{A}_{1}= & -\frac{i \vec{A}_{0}\left(\hat{k} \cdot \vec{\nabla}^{\prime} \epsilon\right)}{\epsilon}-\left(\frac{\vec{\nabla}^{\prime} \epsilon \cdot \vec{\nabla}^{\prime}}{\epsilon}\right) \vec{A}_{0} \\
& -\frac{\vec{\nabla}^{\prime} \epsilon}{\epsilon} \times\left(\vec{\nabla}^{\prime} \times \vec{A}_{0}\right), \\
\nabla^{\prime 2} \vec{A}_{n}+2 i\left(\hat{k} \cdot \vec{\nabla}^{\prime}\right) \vec{A}_{n}+(\epsilon-1) \vec{A}_{n}= & -\frac{i \vec{A}_{n-1}\left(\hat{k} \cdot \vec{\nabla}^{\prime} \epsilon\right)}{\epsilon}-\left(\frac{\vec{\nabla}^{\prime} \epsilon \cdot \vec{\nabla}^{\prime}}{\epsilon}\right) \vec{A}_{n-1} \\
& -\frac{\vec{\nabla}^{\prime} \epsilon}{\epsilon} \times\left(\vec{\nabla}^{\prime} \times \vec{A}_{n-1}\right)-\left(\vec{A}_{n-2} \cdot \vec{\nabla}^{\prime}\right)\left(\frac{\vec{\nabla}^{\prime} \epsilon}{\epsilon}\right) .
\end{aligned}
$$

Let us start with the equation for $A_{0}$. We assume that the frequency $\omega$ of the electromagnetic wave is well above the maximum plasma frequency of the ionosphere. In that case we can solve the equation by using the fact that $\eta=\epsilon-1$ is small. Because the equation is homogeneous, the direction of the vector cannot change as the field propagates, and so we define $\vec{A}_{0}(\vec{x})=\vec{K} e^{a(x)}$, where $\vec{K}$ is a constant vector. Inserting this in the equation for $A_{0}$, we find that

$$
\nabla^{2} a+\vec{\nabla} a \cdot \vec{\nabla} a+2 i \hat{k} \cdot \vec{\nabla} a+\eta=0
$$


where we have dropped the primes to avoid clutter. We look for a solution to this equation in the form of an expansion in powers of $\eta$. Let $a=a_{1}+a_{2}+a_{3}+\ldots$, where $a_{n} \propto \eta^{n}$. Notice that this ordering comes about because $\eta$ is small and has nothing to do with the smallness of $R$. In fact, because $\eta$ is the part of $\epsilon$ which varies, we see that $R$ is proportional to $\nabla \eta$ and is substantially smaller than $\eta$ itself. Because the second term in the equation for $a$ is bilinear, it is of higher order than the other terms. Inserting the expansion in the equation for $a$ and equating terms of the same order yields a hierarchical set of equations for the $a_{j}$, the first three of which are

$$
\begin{aligned}
& \nabla^{2} a_{1}+2 i \hat{k} \cdot \vec{\nabla} a_{1}=-\eta, \\
& \nabla^{2} a_{2}+2 i \hat{k} \cdot \vec{\nabla} a_{2}=-\vec{\nabla} a_{1} \cdot \vec{\nabla} a_{1}, \\
& \nabla^{2} a_{3}+2 i \hat{k} \cdot \vec{\nabla} a_{3}=-2 \vec{\nabla} a_{1} \cdot \vec{\nabla} a_{2} .
\end{aligned}
$$

We solve these equations using Fourier transforms. The Fourier transform $\tilde{a}_{1}$ is

$$
\tilde{a}_{1}(\vec{\xi})=\frac{1}{(2 \pi)^{3 / 2}} \int_{-\infty}^{\infty} a_{1}(\vec{x}) e^{i \vec{\xi} \cdot \vec{x}} d^{3} x
$$

Transforming into $\xi$ space, the equation for $a_{1}$ becomes

$$
-\xi^{2} \tilde{a}_{1}+2 \hat{k} \cdot \vec{\xi} \tilde{a}_{1}=-\frac{1}{(2 \pi)^{3 / 2}} \int_{-\infty}^{\infty} \eta(\vec{x}) e^{i \vec{\xi} \cdot \vec{x}} d^{3} x
$$

Solving for $\tilde{a}_{1}$ and transforming back into $x$ space, we have that

$$
a_{1}(\vec{x})=\frac{1}{(2 \pi)^{3}} \int_{-\infty}^{\infty} d^{3} x^{\prime} \int_{-\infty}^{\infty} d^{3} \xi \frac{\eta\left(\vec{x}^{\prime}\right) e^{i \vec{\xi} \cdot\left(\vec{x}^{\prime}-\vec{x}\right)}}{\xi^{2}-2 \hat{k} \cdot \vec{\xi}}
$$

(The prime in the integrand indicates a dummy variable. All variables are already dimensionless.) We simplify the second integral by changing variables from $\vec{\xi}$ to $\vec{\xi}-\hat{k}$ to obtain

$$
a_{1}(\vec{x})=\frac{1}{(2 \pi)^{3}} \int_{-\infty}^{\infty} d^{3} x^{\prime} \eta\left(\vec{x}^{\prime}\right) e^{i \hat{k} \cdot\left(\vec{x}^{\prime}-\vec{x}\right)} \int_{-\infty}^{\infty} d^{3} \xi \frac{e^{i \vec{\xi} \cdot\left(\vec{x}^{\prime}-\vec{x}\right)}}{\xi^{2}-1}
$$

The integral over $\xi$ may be done analytically:

$$
\begin{aligned}
\int_{-\infty}^{\infty} d^{3} \xi \frac{e^{i \vec{\xi} \cdot\left(\vec{x}^{\prime}-\vec{x}\right)}}{\xi^{2}-1} & =2 \pi \int_{-1}^{1} d \mu \int_{0}^{\infty} d \xi \frac{\xi^{2} e^{i \mu \xi\left|\vec{x}^{\prime}-\vec{x}\right|}}{\xi^{2}-1} \\
& =\frac{4 \pi}{\left|\vec{x}^{\prime}-\vec{x}\right|} \int_{0}^{\infty} d \xi \frac{\xi \sin \left(\xi\left|\vec{x}^{\prime}-\vec{x}\right|\right)}{\xi^{2}-1} \\
& =\frac{2 \pi^{2}}{\left|\vec{x}^{\prime}-\vec{x}\right|} \cos \left(\left|\vec{x}^{\prime}-\vec{x}\right|\right)
\end{aligned}
$$


where the last integral is from Ref. 2, Eq. (3.723.10). Returning to dimensional coordinates the final expression for $a_{1}$ is

$$
a_{1}(\vec{x})=\frac{k^{2}}{4 \pi} \int d^{3} x^{\prime} \eta\left(\vec{x}^{\prime}\right) e^{i \vec{k} \cdot\left(\vec{x}^{\prime}-\vec{x}\right)} \frac{\cos \left(k\left|\vec{x}^{\prime}-\vec{x}\right|\right)}{\left|\vec{x}^{\prime}-\vec{x}\right|} .
$$

The expressions for the $a_{j}$ with higher values of $j$ are of the same form, with $\eta$ replaced by the right-hand side of the equation for $a_{j}$. For $j=2$ we have that

$$
a_{2}(\vec{x})=\frac{1}{4 \pi} \int d^{3} x^{\prime}\left|\vec{\nabla} a_{1}\left(\vec{x}^{\prime}\right)\right|^{2} e^{i \vec{k} \cdot\left(\vec{x}^{\prime}-\vec{x}\right)} \frac{\cos \left(k\left|\vec{x}^{\prime}-\vec{x}\right|\right)}{\left|\vec{x}^{\prime}-\vec{x}\right|},
$$

with similar expressions for $j=3$, etc. Thus we can explicitly solve for $\vec{A}_{0}$ to any desired accuracy in powers of $\eta$.

Now that we have a solution for $\vec{A}_{0}$, we turn to the equation for $\vec{A}_{1}$. We have that

$$
\nabla^{2} \vec{A}_{1}+2 i(\vec{k} \cdot \vec{\nabla}) \vec{A}_{1}+k^{2} \eta \vec{A}_{1}=-\vec{S}_{0}
$$

where the source term $\vec{S}_{0}$ is

$$
\vec{S}_{0}=\frac{i \vec{A}_{0}(\vec{k} \cdot \vec{\nabla} \epsilon)}{\epsilon}+\left(\frac{\vec{\nabla} \epsilon \cdot \vec{\nabla}}{\epsilon}\right) \vec{A}_{0}+\frac{\vec{\nabla} \epsilon}{\epsilon} \times\left(\vec{\nabla} \times \vec{A}_{0}\right) .
$$

We have expressed $\vec{A}_{0}$ in the form $\vec{A}_{0}=\vec{K} e^{a}$. It is convenient to work with $\vec{B}_{1}=\vec{A}_{1} /\left|A_{0}\right|$. The equation for $\vec{B}_{1}$ is

$$
\nabla^{2} \vec{B}_{1}+2 i(\vec{k} \cdot \vec{\nabla}) \vec{B}_{1}+2(\vec{\nabla} a \cdot \vec{\nabla}) \vec{B}_{1}=-\vec{S}_{0}^{\prime}
$$

where

$$
\vec{S}_{0}^{\prime}=\frac{i \hat{K}(\vec{k} \cdot \vec{\nabla} \epsilon)+\vec{\nabla} a(\hat{K} \cdot \vec{\nabla} \epsilon)}{\epsilon}
$$

and $\hat{K}=\vec{K} /|K|$. The equation for $\vec{B}_{1}$ is not homogeneous: the source term has a part parallel to $\vec{A}_{0}$ and a part which is not, so this term leads to polarization mixing as the wave propagates through the ionosphere.

The source term $S_{0}^{\prime}$ will contain parts proportional to various powers of $\eta$, beginning with the first power, and it may be expanded in the form

$$
\vec{S}_{0}^{\prime}=\vec{S}_{0}^{(1)}+\vec{S}_{0}^{(2)}+\ldots
$$

where the term with superscript $n$ is proportional to $\eta^{n}$. We may expand $\vec{B}_{1}$ in the same way. The term $\vec{\nabla} a=\vec{\nabla}\left(a_{1}+a_{2}+a_{3}+\ldots\right)$ is also a series of terms proportional to $\eta, \eta^{2}$, etc. Inserting these expansions into the equation for $\vec{B}_{1}$ and equating terms with the same power of $\eta$, we have that

$$
\nabla^{2} \vec{B}_{1}^{(n)}+2 i(\vec{k} \cdot \vec{\nabla}) \vec{B}_{1}^{(n)}=-S_{0}^{(n)}-2 \sum_{m=1}^{n-1}\left(\vec{\nabla} a_{m} \cdot \vec{\nabla}\right) \vec{B}_{1}^{(n-m)}
$$


This is the same type of equation as we found for the $a_{j}$, and we can solve it in the same manner as before. We find that

$$
\vec{B}_{1}^{(1)}(\vec{x})=\frac{1}{4 \pi} \int d^{3} x^{\prime} \vec{S}_{0}^{(1)}\left(\vec{x}^{\prime}\right) e^{i \vec{k} \cdot\left(\vec{x}^{\prime}-\vec{x}\right)} \frac{\cos \left(k\left|\vec{x}^{\prime}-\vec{x}\right|\right)}{\left|\vec{x}^{\prime}-\vec{x}\right|}
$$

and

$$
\vec{B}_{1}^{(n)}(\vec{x})=\frac{1}{4 \pi} \int d^{3} x^{\prime}\left[S_{0}^{(n)}\left(\vec{x}^{\prime}\right)-2 \sum_{m=1}^{n-1}\left(\vec{\nabla} a_{m} \cdot \vec{\nabla}\right) \vec{B}_{1}^{(n-m)}\left(\vec{x}^{\prime}\right)\right] e^{i \vec{k} \cdot\left(\vec{x}^{\prime}-\vec{x}\right)} \frac{\cos \left(k\left|\vec{x}^{\prime}-\vec{x}\right|\right)}{\left|\vec{x}^{\prime}-\vec{x}\right|}
$$

Once again this gives an explicit construction for calculating $\vec{B}_{1}$, and therefore $\vec{A}_{1}$, to any desired accuracy in powers of $\eta$.

Solutions for $\vec{A}_{2}, \vec{A}_{3}$, etc. may be found in the same manner. We shall not give the expressions here. As for $A_{1}$ it is easier to work with the quantities $\vec{B}_{n}=\vec{A}_{n} /\left|A_{0}\right|$.

\section{Solutions for Laminar Ionosphere with Waves}

In the last section we derived a general solution for the electric field of an electromagnetic wave propagating through a medium with a slowly varying dielectric function. In this section we will find the solution for the special case where the dielectric function $\epsilon$ has an arbitrary profile in the $\hat{z}$ direction (as long as the peak value of $\epsilon-1 \ll 1$ ), with large-wavelength harmonic waves superimposed on this profile. We take the dielectric function to be

$$
\epsilon(\vec{x})=1+\sum_{j} \eta_{j}(z) e^{i \vec{x} \cdot \vec{\Lambda}_{j}}
$$

where $\eta_{j} \ll 1$ and $\left|\Lambda_{j}\right| \ll k$ for all $j$.

We consider first the expression for $a_{1}$. We may rewrite Eq. 20 as

$$
a_{1}(\vec{x})=\frac{k^{2}}{4 \pi} \int d^{3} x^{\prime} \eta\left(\vec{x}^{\prime}+\vec{x}\right) e^{i \vec{k} \cdot \vec{x}^{\prime}} \frac{\cos \left(k\left|\vec{x}^{\prime}\right|\right)}{\left|\vec{x}^{\prime}\right|}
$$

Plugging in our expression for $\eta$, we have

$$
\begin{aligned}
a_{1}(\vec{x}) & =\frac{k^{2}}{4 \pi} \sum_{j} e^{i \vec{x} \cdot \vec{\Lambda}_{j}} \int d^{3} x^{\prime} \eta_{j}\left(z^{\prime}+z\right) e^{i\left(\vec{k}+\vec{\Lambda}_{j}\right) \cdot \vec{x}^{\prime}} \frac{\cos \left(k\left|\vec{x}^{\prime}\right|\right)}{\left|\vec{x}^{\prime}\right|} \\
& =\frac{k^{2}}{4 \pi} \sum_{j} e^{i \vec{x} \cdot \vec{\Lambda}_{j}} \int_{-\infty}^{\infty} d z^{\prime} \eta_{j}\left(z^{\prime}+z\right) e^{i\left(k_{z}+\Lambda_{j_{z}}\right) z^{\prime}}
\end{aligned}
$$




$$
\begin{aligned}
& \times \int_{-\infty}^{\infty} d x^{\prime} \int_{-\infty}^{\infty} d y^{\prime} e^{i\left(k_{x}+\Lambda_{j_{x}}\right) x^{\prime}} e^{i\left(k_{y}+\Lambda_{j_{y}}\right) y^{\prime}} \frac{\cos \left(k \sqrt{x^{\prime 2}+y^{\prime 2}+z^{\prime 2}}\right)}{\sqrt{x^{\prime 2}+y^{\prime 2}+z^{\prime 2}}} \\
= & \frac{k^{2}}{4 \pi} \sum_{j} e^{i \vec{x} \cdot \vec{\Lambda}_{j}} \int_{-\infty}^{\infty} d z^{\prime} \eta_{j}\left(z^{\prime}+z\right) e^{i\left(k_{z}+\Lambda_{j_{z}}\right) z^{\prime}} I_{j}\left(z^{\prime}, \vec{k}, \vec{\Lambda}_{j}\right)
\end{aligned}
$$

where $I_{j}$ is the integral over $x^{\prime}$ and $y^{\prime}$. Define $M_{j}^{2}=k^{2}-\left(k_{x}+\Lambda_{j_{x}}\right)^{2}-\left(k_{y}+\Lambda_{j_{y}}\right)^{2}$. Switching to polar coordinates, $I_{j}$ may be written as

$$
\begin{aligned}
I_{j}\left(z^{\prime}, \vec{k}, \vec{\Lambda}_{j}\right) & =\int_{-\pi}^{\pi} d \phi \int_{0}^{\infty} d r^{\prime} r^{\prime} \frac{e^{i \sqrt{k^{2}-M_{j}^{2}} r^{\prime} \cos \phi} \cos \left(k \sqrt{r^{\prime 2}+z^{\prime 2}}\right)}{\sqrt{r^{\prime 2}+z^{\prime 2}}} \\
& =2 \pi \int_{0}^{\infty} d r^{\prime} r^{\prime} J_{0}\left(\sqrt{k^{2}-M_{j}^{2}} r^{\prime}\right) \frac{\cos \left(k \sqrt{r^{\prime 2}+z^{\prime 2}}\right)}{\sqrt{r^{\prime 2}+z^{\prime 2}}}
\end{aligned}
$$

where $J_{0}$ is a Bessel function and the integral was obtained from Ref. 2 [see Eqs. (3.715.13) and (3.715.18)]. We change variables to $q=\sqrt{r^{12}+z^{\prime 2}}$ and use Ref. 2, Eq. (6.677.2) to obtain

$$
\begin{aligned}
I_{j}\left(z^{\prime}, \vec{k}, \vec{\Lambda}_{j}\right) & =2 \pi \int_{\left|z^{\prime}\right|}^{\infty} d q J_{0}\left(\sqrt{k^{2}-M_{j}^{2}} \sqrt{q^{2}-z^{\prime 2}}\right) \cos (k q) \\
& =-2 \pi \frac{e^{\left|z^{\prime}\right|\left|M_{j}\right|}}{\left|M_{j}\right|} \quad\left(M_{j}^{2}<0\right) \\
& =-2 \pi \frac{\sin \left(\left|z^{\prime}\right| M_{j}\right)}{M_{j}} \quad\left(M_{j}^{2}>0\right) .
\end{aligned}
$$

We may write $M_{j}^{2}$ in the form $M_{j}^{2}=k_{z}^{2}-2 k_{x} \Lambda_{j_{x}}-\Lambda_{j_{x}}^{2}-2 k_{y} \Lambda_{j_{y}}-\Lambda_{j_{y}}^{2}$. Recalling that $k_{z}>0$ we see that if there were no horizontal gradients in the ionosphere $(\vec{\Lambda}=0) M^{2}$ would always be greater than zero. In that case $I$ is bounded and waves can propagate. When horizontal gradients are present, there are values of $k_{z}$ for which $M^{2}<0$, in which case $I \rightarrow-\infty$ and the electric field is exponentially damped as the altitude increases. Because we are assuming that $|\Lambda| \ll k$, this will only happen for waves launched at angles very close to the horizon, and we shall not be concerned with this case.

Assuming $M^{2}>0$ the final expression for $a_{1}$ is

$$
a_{1}(\vec{x})=\frac{-k^{2}}{2} \sum_{j} \frac{e^{i \vec{x} \cdot \vec{\Lambda}_{j}}}{M_{j}} \int_{-\infty}^{\infty} d z^{\prime} \eta_{j}\left(z^{\prime}\right) \sin \left(\left|z^{\prime}-z\right| M_{j}\right) e^{i\left(k_{z}+\Lambda_{j_{z}}\right)\left(z^{\prime}-z\right)} .
$$

This solution does not meet the boundary conditions of the problem. The condition for the forwardgoing part of the wave will be met if $\vec{K}=\vec{E}_{0}$ and $a_{1}=0$ at $z=-\infty$. This may be accomplished by adding a solution of the associated homogeneous equation to the special solution we have derived. 
The homogeneous equation is the equation for $a_{1}$ with $\eta=0$, and the required solution $h_{1}$ of this equation is

$$
h_{1}(\vec{x})=\frac{-k^{2}}{2} \sum_{j} \frac{e^{i \vec{x} \cdot \vec{\Lambda}_{j}}}{M_{j}} \int_{-\infty}^{\infty} d z^{\prime} \eta_{j}\left(z^{\prime}\right) \sin \left(\left(z^{\prime}-z\right) M_{j}\right) e^{i\left(k_{z}+\Lambda_{j z}\right)\left(z^{\prime}-z\right)} .
$$

Subtracting $h_{1}$ from the special solution yields the solution $a_{1}$ with the desired behavior:

$$
a_{1}(\vec{x})=-k^{2} \sum_{j} \frac{e^{i \vec{x} \cdot \vec{\Lambda}_{j}}}{M_{j}} \int_{-\infty}^{z} d z^{\prime} \eta_{j}\left(z^{\prime}\right) \sin \left(\left(z-z^{\prime}\right) M_{j}\right) e^{-i\left(k_{z}+\Lambda_{j_{z}}\right)\left(z-z^{\prime}\right)} .
$$

The above expression for $a_{1}$ contains both forward- and backward-going waves. To see this we rewrite the sine function in the integrand as the difference of two complex exponentials. Inserting this form in Eq. 37, we have that

$$
a_{1}(\vec{x})=\frac{i k^{2}}{2} \sum_{j} \frac{e^{i \vec{x} \cdot \vec{\Lambda}_{j}}}{M_{j}} \int_{-\infty}^{z} d z^{\prime} \eta_{j}\left(z^{\prime}\right) e^{-i\left(k_{z}+\Lambda_{j z}\right)\left(z-z^{\prime}\right)}\left[e^{i\left(z-z^{\prime}\right) M_{j}}-e^{-i\left(z-z^{\prime}\right) M_{j}}\right] .
$$

In order to understand the behavior of the integrand, we expand $M_{j}$ in powers of $\Lambda_{j} / k_{z}$. Expanding to first order we have that

$$
\begin{aligned}
M_{j} & =\sqrt{k_{z}^{2}-2 k_{x} \Lambda_{j_{x}}-2 k_{y} \Lambda_{j_{y}}-\Lambda_{j_{x}}^{2}-\Lambda_{j_{y}}^{2}} \\
& \approx k_{z}-\frac{k_{x} \Lambda_{j_{x}}}{k_{z}}-\frac{k_{y} \Lambda_{j_{y}}}{k_{z}} .
\end{aligned}
$$

Using the expansion for $M_{j}$, the integrand of the equation for $a_{1}$ becomes

$$
\begin{aligned}
& \eta_{j}\left(z^{\prime}\right) e^{-i\left(k_{z}+\Lambda_{j_{z}}\right)\left(z-z^{\prime}\right)}\left[e^{i\left(z-z^{\prime}\right) M_{j}}-e^{-i\left(z-z^{\prime}\right) M_{j}}\right] \approx \\
& \eta_{j}\left(z^{\prime}\right)\left[e^{-i\left(z-z^{\prime}\right) \vec{k} \cdot \vec{\Lambda}_{j} / k_{z}}-e^{-2 i\left(z-z^{\prime}\right) k_{z}} e^{i\left(z-z^{\prime}\right) \vec{k} \cdot \vec{\Lambda}_{j} / k_{z}}\right] .
\end{aligned}
$$

The second term has a complex exponential factor $e^{-2 i\left(z-z^{\prime}\right) k_{z}}$ which oscillates very rapidly compared to the rate of change of the rest of the integrand. The average value of the rapidly varying factor is zero, so the value of the integral is quite small. Thus we may write $a_{1}$ as

$$
a_{1}=a_{1}^{+}+\delta e^{-2 i z k_{z}}
$$

where $a_{1}^{+}$and $\delta$ are the integrals of the first and second terms, respectively, and $\delta<<a_{1}^{+}$. Recalling the expansion of the electric field, we see that

$$
\begin{aligned}
\vec{E} & =\vec{E}_{0} e^{i \vec{k} \cdot \vec{x}} e^{a_{1}} \\
& \approx \vec{E}_{0} e^{i \vec{k} \cdot \vec{x}} e^{a_{1}^{+}}\left(1+\delta e^{-2 i z k_{z}}\right) \\
& =\vec{E}_{0} e^{i\left(x k_{x}+y k_{y}+z k_{z}\right)+a_{1}^{+}}+\delta \vec{E}_{0} e^{i\left(x k_{x}+y k_{y}-z k_{z}\right)}
\end{aligned}
$$


The term proportional to $\delta$ is the backward-going wave and is very small compared to the forwardgoing wave. To be perfectly correct this term should be removed by including an appropriate backward-going component in the initial wave at $z=-\infty$. This can be done, with further corrections to be made at higher orders in the expansion, but because this term is so small we shall simply drop it in what follows. The backward-going wave can, in principle, contribute to the forward-going wave amplitude at higher orders because of multiple scattering processes, in which the backwardgoing wave interacts with the ionosphere and produces a small forward-going component, but these terms will be of order $\delta^{2}$ and higher and will be negligible compared to the direct forward-going component.

Dropping the backward-going wave, our final expression for $a_{1}$ is

$$
a_{1}(\vec{x})=\frac{i k^{2}}{2} \sum_{j} \frac{e^{i \vec{x} \cdot \vec{\Lambda}_{j}}}{M_{j}} \int_{-\infty}^{z} d z^{\prime} \eta_{j}\left(z^{\prime}\right) e^{i\left(M_{j}-k_{z}-\Lambda_{j z}\right)\left(z-z^{\prime}\right)} .
$$

This expression for $a_{1}$ is a sum of functions of $z$ times complex exponentials. The integrands of the integrals to be evaluated for the other $a_{j}$ and for the $\vec{B}_{n}^{(j)}$ will also have this form because these integrands are composed of sums and products of the $a_{j}$, derivatives of the $a_{j}$, and gradients of the dielectric function. We may therefore apply the same method as above to evaluate all of the integrals. Because the integrand for the $a_{n}$ integral involves products of the $a_{j}$ with $j<n$, wave vectors which are sums and differences of the original $\vec{\Lambda}_{j}$ will appear at higher orders in the expansion for $a$.

The boundary conditions of the problem will be met if all of the $a_{j}$ and $\vec{A}_{n}^{(j)}$ are zero at $z=-\infty$ for $j$ and $n \geq 1$. This may be accomplished by adding solutions of the associated homogeneous equations to the special solutions we have derived for these quantities, as shown above for $a_{1}$. For each equation the special solution $f$ is of the generic form

$$
f(\vec{x})=-\frac{1}{2} \sum_{j} \frac{e^{i \vec{x} \cdot \vec{V}_{j}}}{M_{j}} \int_{-\infty}^{\infty} d z^{\prime} g\left(z^{\prime}\right) \sin \left(\left|z^{\prime}-z\right| M_{j}\right) e^{i\left(k_{z}+V_{j_{z}}\right)\left(z^{\prime}-z\right)}
$$

where $\vec{V}$ is some combination of the $\vec{\Lambda}_{j}$ and $M^{2}=k_{z}^{2}-2 k_{x} V_{j_{x}}-V_{j_{x}}^{2}-2 k_{y} V_{j_{y}}-V_{j_{y}}^{2}$. A solution $h$ of the associated homogeneous equation is

$$
h(\vec{x})=-\frac{1}{2} \sum_{j} \frac{e^{i \vec{x} \cdot \vec{V}_{j}}}{M_{j}} \int_{-\infty}^{\infty} d z^{\prime} g\left(z^{\prime}\right) \sin \left(\left(z^{\prime}-z\right) M_{j}\right) e^{i\left(k_{z}+V_{j_{z}}\right)\left(z^{\prime}-z\right)} .
$$

Subtracting $h$ from the special solution yields the solution $f$ with the desired boundary conditions:

$$
f(\vec{x})=-\sum_{j} \frac{e^{i \vec{x} \cdot \vec{V}_{j}}}{M_{j}} \int_{-\infty}^{z} d z^{\prime} g\left(z^{\prime}\right) \sin \left[\left(z-z^{\prime}\right) M_{j}\right] e^{-i\left(k_{z}+V_{j z}\right)\left(z-z^{\prime}\right)}
$$


As before, removing the backward-going components yields

$$
f(\vec{x})=\frac{i}{2} \sum_{j} \frac{e^{i \vec{x} \cdot \vec{V}_{j}}}{M_{j}} \int_{-\infty}^{z} d z^{\prime} g\left(z^{\prime}\right) e^{i\left(M_{j}-k_{z}-V_{j z}\right)\left(z-z^{\prime}\right)}
$$

As an example we derive the solutions for $a_{2}$ and $\vec{B}_{1}^{(1)}$. The expression for $a_{2}$ involves the gradient of $a_{1}$. To simplify the notation we define

$$
\gamma_{j}(z)=-\frac{k^{2}}{M_{j}} \int_{-\infty}^{z} d z^{\prime} \eta_{j}\left(z^{\prime}\right) e^{i\left(M_{j}-k_{z}-\Lambda_{j_{z}}\right)\left(z-z^{\prime}\right)}
$$

in which case we have

$$
a_{1}(\vec{x})=\sum_{j} e^{i \vec{x} \cdot \vec{\Lambda}_{j}} \gamma_{j}(z)
$$

The gradient of $a_{1}$ is then

$$
\vec{\nabla} a_{1}=\sum_{j} e^{i \vec{x} \cdot \vec{\Lambda}_{j}}\left(i \gamma_{j} \dot{\vec{\Lambda}}_{j}+\hat{z} \frac{d \gamma_{j}}{d z}\right)
$$

The general expression for $a_{2}$ is given in Eq. 21 and involves $\vec{\nabla} a_{1} \cdot \vec{\nabla} a_{1}$. In the case of a laminar ionosphere with waves, this product is

$$
\vec{\nabla} a_{1} \cdot \vec{\nabla} a_{1}=\sum_{j} \sum_{l} e^{i \vec{x} \cdot\left(\vec{\Lambda}_{j}+\vec{\Lambda}_{l}\right)}\left(-\gamma_{j} \gamma_{l} \vec{\Lambda}_{j} \cdot \vec{\Lambda}_{l}+i \gamma_{j} \Lambda_{j_{z}} \frac{d \gamma_{l}}{d z}+i \gamma_{l} \Lambda_{l z} \frac{d \gamma_{j}}{d z}+\frac{d \gamma_{j}}{d z} \frac{d \gamma_{l}}{d z}\right) .
$$

This expression is a sum of complex exponentials times functions of $z$, as claimed above, and we can therefore write the solution for $a_{2}$ as

$$
\begin{aligned}
a_{2}= & \frac{i}{2} \sum_{j} \sum_{l} \frac{e^{i \vec{x} \cdot\left(\vec{\Lambda}_{j}+\vec{\Lambda}_{l}\right)}}{M_{j l}} \int_{-\infty}^{z} d z^{\prime}\left(-\gamma_{j} \gamma_{l} \vec{\Lambda}_{j} \cdot \vec{\Lambda}_{l}+i \gamma_{j} \Lambda_{j z} \frac{d \gamma_{l}}{d z}\right. \\
& \left.+i \gamma_{l} \Lambda_{l_{z}} \frac{d \gamma_{j}}{d z}+\frac{d \gamma_{j}}{d z} \frac{d \gamma_{l}}{d z}\right) e^{i\left(M_{j l}-k_{z}-\Lambda_{j z}-\Lambda_{l_{z}}\right)\left(z-z^{\prime}\right)}
\end{aligned}
$$

where $M_{j l}$ is given by the general expression for $M$ stated above with $\vec{V}=\vec{\Lambda}_{j}+\vec{\Lambda}_{l}$.

The general solution for $\vec{B}_{1}^{(1)}$ is given by Eq. 28 . The source term is given by $\vec{S}_{0}^{(1)}=i \hat{E}_{0}\left(\vec{k}_{0} \cdot \vec{\nabla} \epsilon\right)$, which for our laminar model ionosphere with waves is

$$
\vec{S}_{0}^{(1)}=i \hat{E}_{0} \sum_{j} e^{i \vec{x} \cdot \vec{\Lambda}_{j}}\left(i \vec{k} \cdot \vec{\Lambda}_{j} \eta_{j}+\vec{k}_{z} \frac{d \eta_{j}}{d z}\right)
$$


This is once again in the desired form, and we see that

$$
\vec{B}_{1}^{(1)}(\vec{x})=-\frac{i \hat{E}_{0}}{2} \sum_{j} \frac{e^{i \vec{x} \cdot \vec{\Lambda}_{j}}}{M_{j}} \int_{-\infty}^{z} d z^{\prime}\left(\vec{k} \cdot \vec{\Lambda}_{j} \eta_{j}-i \vec{k}_{z} \frac{d \eta_{j}}{d z}\right) e^{i\left(M_{j}-k_{z}-\Lambda_{j z}\right)\left(z-z^{\prime}\right)} .
$$

The vector in this term is parallel to the original field, so it does not introduce any polarization mixing. This is not true at higher orders.

\section{Definition of the Local Wave Vector and Calculation of the Ray Path}

We have developed a solution for the case where the initial field is a plane wave with wave vector $\vec{k}$. Now consider starting at the point $\vec{x}_{0}$ and following the progress of a point of constant phase on the wave as it propagates upwards through the ionosphere. Variations in the electron density (and therefore the dielectric function) will cause the wavefront to be tilted from its original orientation as it moves, i.e., refracted. We wish to calculate the path followed by a point of constant phase, which we shall call the ray path.

The first step is to define the local wave vector $\vec{\kappa}(\vec{x})$. Our expression for the electric field is complex and may be written in the form $E=T e^{i \Phi}$, where $T$ is the amplitude of the field and $\Phi$ is the phase. The wave vector of a field is strictly defined only in the case for which $T$ is a constant and $\Phi$ is linear in $\vec{x}$, in which case the wave vector is the gradient of the phase. In this problem the field does not meet these conditions, but it is true that the variations of the field are caused by variations in the electron density which occur on a scale large compared to the wavelength of the radiation. Thus, locally the field will resemble a plane wave, although over large distances there may be significant deviations from planarity. The orientation of the quasi plane wave at any point may be found from a Taylor expansion of the phase at that point. The constant term in the expansion is the total phase accumulated by the wave in propagating to that point, and we define the coefficient of the linear term as the local wave vector. Thus we have

$$
\vec{\kappa}(\vec{x})=\vec{\nabla} \Phi(\vec{x}) \text {. }
$$

Using our expression for the electric field and ignoring the polarization mixing terms $\vec{B}_{j}$, we have that

$$
\vec{\kappa}(\vec{x})=\vec{k}+\operatorname{Im}(\vec{\nabla} a)
$$

To check the interpretation of $\vec{\kappa}$ as a wave vector, we calculate its norm. Using the equation for $a$, we find that

$$
\begin{aligned}
\kappa^{2} & =k^{2}+2 \operatorname{Im}(\vec{k} \cdot \vec{\nabla} a)+[\operatorname{Im}(\nabla a)]^{2} \\
& =(1+\eta) k^{2}+\operatorname{Re}\left(\nabla^{2} a\right)+[\operatorname{Re}(\nabla a)]^{2}
\end{aligned}
$$


In a lossless medium $[\operatorname{Re}(a)=0]$, this reduces to Snell's law.

Given the local wave vector we can calculate the ray path $\vec{X}$. We express this by giving the horizontal displacements $X$ and $Y$ as functions of the altitude $z$. The local wave vector gives the direction of motion at each point:

$$
\begin{aligned}
& \frac{d X}{d z}=\frac{\kappa_{x}}{\kappa_{z}}=\frac{k_{x}+\operatorname{Im}\left(\partial_{x} a\right)}{k_{z}+\operatorname{Im}\left(\partial_{z} a\right)}, \\
& \frac{d Y}{d z}=\frac{\kappa_{x}}{\kappa_{z}}=\frac{k_{y}+\operatorname{Im}\left(\partial_{y} a\right)}{k_{z}+\operatorname{Im}\left(\partial_{z} a\right)},
\end{aligned}
$$

with the initial conditions $X\left(z_{0}\right)=x_{0}, Y\left(z_{0}\right)=y_{0}$. Expanding to first order we have that

$$
\begin{aligned}
& \frac{d X}{d z} \approx \frac{k_{x}}{k_{z}}\left[1+\frac{\operatorname{Im}\left(\partial_{x} a_{1}\right)}{k_{x}}-\frac{\operatorname{Im}\left(\partial_{z} a_{1}\right)}{k_{z}}\right], \\
& \frac{d Y}{d z} \approx \frac{k_{y}}{k_{z}}\left[1+\frac{\operatorname{Im}\left(\partial_{y} a_{1}\right)}{k_{y}}-\frac{\operatorname{Im}\left(\partial_{z} a_{1}\right)}{k_{z}}\right] .
\end{aligned}
$$

The zeroth-order ray path is a straight line; we begin to see the effects of the ionosphere at first order. For the case of a stratified ionosphere with waves, we have, to first order,

$$
\begin{aligned}
& \frac{d X}{d z}=\frac{k_{x}}{k_{z}}+\operatorname{Re} \sum_{j} e^{i \vec{X} \cdot \vec{\Lambda}_{j}}\left[\left(\frac{\Lambda_{x}}{k_{z}}+\frac{k_{x}}{k_{z}}-\frac{k_{x} M_{j}}{k_{z}^{2}}\right) \gamma_{j}-\frac{\eta_{j} k_{x} k^{2}}{2 k_{z}^{2} M_{j}}\right], \\
& \frac{d Y}{d z}=\frac{k_{y}}{k_{z}}+\operatorname{Re} \sum_{j} e^{i \vec{X} \cdot \vec{\Lambda}_{j}}\left[\left(\frac{\Lambda_{y}}{k_{z}}+\frac{k_{y}}{k_{z}}-\frac{k_{y} M_{j}}{k_{z}^{2}}\right) \gamma_{j}-\frac{\eta_{j} k_{y} k^{2}}{2 k_{z}^{2} M_{j}}\right] .
\end{aligned}
$$

These equations must in general be solved numerically.

The equations for the ray path are not invariant under changes in the size of $k$. In addition to the explicit $k$ dependence in the above expressions, there is an implicit dependence through the functions $\gamma_{j}$ and $\eta_{j}$. The variation with $k$ means that the ray path for a given ionosphere, initial launch direction, and location $\vec{x}_{0}$ is frequency dependent. It is often of interest to measure the field at a detection location $\vec{x}_{1}$ produced by a source at location $\vec{x}_{0}$. In order for rays of different frequencies to arrive at the same detector location, they must leave the source at different angles. We will calculate the emission angle necessary to arrive at a given detector location as a function of frequency.

The effect of the ionosphere on the ray paths decreases with increasing frequency; for extremely high frequencies the rays follow straight lines. The straight-line path $\vec{X}_{\infty}$ followed by extremely high-frequency rays is

$$
X_{\infty}(z)=x_{0}+\left(z-z_{0}\right) \frac{k_{x}}{k_{z}}
$$




$$
\begin{aligned}
Y_{\infty}(z) & =y_{0}+\left(z-z_{0}\right) \frac{k_{y}}{k_{z}} \\
Z_{\infty} & =z .
\end{aligned}
$$

The true path followed by a ray of frequency $f$ will not differ greatly from the straight-line path, so we will characterize it by small deviations $\vec{\Delta}$ from the straight-line path:

$$
\begin{aligned}
& \Delta_{x}(z) \equiv X(z)-X_{\infty}(z)=X(z)-x_{0}-\left(z-z_{0}\right) k_{x} / k_{z} \\
& \Delta_{y}(z) \equiv Y(z)-Y_{\infty}(z)=Y(z)-y_{0}-\left(z-z_{0}\right) k_{y} / k_{z} \\
& \Delta_{z}(z) \equiv 0
\end{aligned}
$$

In order to arrive at the correct endpoint, the ray of frequency $f$ must leave the source with a wave vector $\vec{k}^{\prime}$ slightly different from $\vec{k}$. Wave vector $\vec{k}^{\prime}$ has the same magnitude $k$ as the wave vector in the straight-line direction but is oriented differently. Because we require the true ray path $\vec{X}\left(k^{\prime}\right)$ to intersect the straight-line ray path $\vec{X}_{\infty}(k)$ at $z=z_{1}$, we have that

$$
\begin{aligned}
\left(z_{1}-z_{0}\right) \frac{k_{x}^{\prime}}{k_{z}^{\prime}}+\Delta_{x}\left(k^{\prime}\right) & =\left(z_{1}-z_{0}\right) \frac{k_{x}}{k_{z}} \\
\left(z_{1}-z_{0}\right) \frac{k_{y}^{\prime}}{k_{z}^{\prime}}+\Delta_{y}\left(k^{\prime}\right) & =\left(z_{1}-z_{0}\right) \frac{k_{y}}{k_{z}} \\
\sqrt{k_{x}^{\prime 2}+k_{y}^{\prime 2}+k_{z}^{\prime 2}} & =k .
\end{aligned}
$$

We solve these equations for $\vec{k}^{\prime}$ to obtain

$$
\vec{k}^{\prime}=k \frac{\vec{k}+\vec{\Delta}_{r}^{\prime} k_{z}}{\left|\vec{k}+\vec{\Delta}_{r}^{\prime} k_{z}\right|},
$$

where $\vec{\Delta}_{r}^{\prime}=\vec{\Delta}\left(k^{\prime}\right) /\left(z_{1}-z_{0}\right)$, the horizontal deviation of the true path with wave vector $\vec{k}^{\prime}$ from the straight-line ray path divided by the altitude of the receiver. We note that $\Delta_{r}^{\prime}$ decreases and $\vec{k}^{\prime}$ approaches $\vec{k}$ for a given $\vec{\Delta}$ as the altitude of the receiver increases, reflecting the fact that a given horizontal displacement can be achieved with a smaller angle change if the lever arm is longer.

The expression for $\vec{k}^{\prime}$ in Eq. 64 is implicit because $\vec{\Delta}_{r}^{\prime}$ depends on $\vec{k}^{\prime}$. However, because $\vec{\Delta}_{r}^{\prime}$ is small and $\vec{k}^{\prime}$ differs only slightly from $\vec{k}$, we may solve the equation iteratively. Thus we get the first-order expression for $\vec{k}^{\prime}$ by evaluating $\vec{\Delta}_{r}$ for $\vec{k}$. We then use this result to reevaluate $\Delta_{r}^{\prime}$, which can be used to obtain the second-order correction to $\vec{k}^{\prime}$, and so on.

We define $\delta \vec{k}$ as the difference between $\vec{k}$ and $\vec{k}^{\prime}$. We see that

$$
\delta \vec{k}=k_{z}\left[\hat{k}\left(\hat{k} \cdot \vec{\Delta}_{r}\right)-\vec{\Delta}_{r}\right]
$$


The difference vector is almost orthogonal to $\vec{k}$. The dot product is

$$
\delta \vec{k} \cdot \vec{k}=k \frac{k^{2}+\vec{k} \cdot \vec{\Delta}_{r} k_{z}}{\sqrt{k^{2}+2 \vec{k} \cdot \vec{\Delta}_{r} k_{z}+\Delta_{r}^{2} k_{z}^{2}}}-k^{2}
$$

If we expand the square root in powers of $\vec{\Delta}_{r}$, we see the zeroth- and first-order terms in the above expression cancel, leaving only-second order terms and higher.

\section{Refractive Effects on Phase}

$\mathrm{Up}_{\mathrm{p}}$ to this point we have calculated the changes in the phase and amplitude of a signal with a given $\vec{k}$. We showed in the last section that signals of different frequencies must have wave vectors oriented in different directions in order to start from a common source and arrive at the same point after propagating through the ionosphere. Strictly speaking, this means that after determining the change in the wave vector for each frequency we should go back and recalculate $a$ for the new wave vector, obtain the new ray path, find a corrected wave vector if the ray does not arrive at the correct detector location, and continue iterating this process until the ray ends up sufficiently close to the detector. In fact the deviations of the different rays are relatively small, and it is generally sufficient to approximate the changes in $a$ caused by refraction by using a first-order Taylor expansion in $\vec{k}$. The phase without refractive effects is

$$
\Phi=\vec{k} \cdot \vec{x}+\operatorname{Im}[a(\vec{x}, \vec{k})] .
$$

When refractive effects are included, we have that

$$
\begin{aligned}
\Phi & =\vec{k}^{\prime} \cdot \vec{x}+\operatorname{Im}\left[a\left(\vec{x}, \vec{k}^{\prime}\right)\right] \\
& \approx \vec{k} \cdot \vec{x}+\operatorname{Im}[a(\vec{x}, \vec{k})]+\delta \vec{k} \cdot \vec{x}+\delta \vec{k} \cdot \operatorname{Im}\left[\vec{\nabla}_{k} a(\vec{x}, \vec{k})\right] .
\end{aligned}
$$

Because $\vec{k}$ is the wave vector for the straight-line path, we see that $\left(\vec{x}-\vec{x}_{0}\right) \| \vec{k}$. We showed in the last section that $\delta \vec{k}$ is orthogonal to $\vec{k}$ and therefore to $\vec{x}-\vec{x}_{0}$ up to second order, so refractive effects first appear in the phase at second order. The second-order expression for the phase is

$$
\Phi=\vec{k} \cdot \vec{x}+\operatorname{Im}\left[a_{1}\right]+\operatorname{Im}\left[a_{2}\right]+\delta \vec{k} \cdot \vec{x}+\delta \vec{k} \cdot \operatorname{Im}\left[\vec{\nabla}_{k} a_{1}\right]
$$

where $\delta \vec{k}$ is the first-order change in the wave vector and $\vec{\nabla}_{k}$ is the gradient operator in $k$ space. The gradient of $a_{1}$ is messy but straightforward to calculate. We assume that the $k$ dependence 
of $\eta$ can be factored, so that $k^{2} \eta(z, \vec{k})=N(\vec{k}) \hat{\eta}(z)$. Using the approximate form for $a_{1}$, where $\sin \left[\left(z-z^{\prime}\right) M\right]$ is replaced by $-i e^{i\left(z-z^{\prime}\right) M} / 2$, we find that

$$
\begin{aligned}
\vec{\nabla}_{k} a_{1}= & \frac{i}{2} \sum_{j} \frac{e^{i \vec{x} \cdot \vec{\Lambda}_{j}}}{M_{j}}\left\{\left(\vec{\nabla}_{k} N+\frac{N}{M_{j}^{2}}\left[\vec{\Lambda}_{j}-\left(k_{z}+\Lambda_{j_{z}}\right) \hat{z}\right]\right) \int_{-\infty}^{z} d z^{\prime} \hat{\eta}_{j}\left(z^{\prime}\right) e^{i\left(M_{j}-k_{z}-\Lambda_{j z}\right)\left(z-z^{\prime}\right)}(70)\right. \\
& \left.\left.-i \frac{N}{M_{j}}\left[\vec{\Lambda}_{j}+\left(M_{j}-k_{z}-\Lambda_{j_{z}}\right) \hat{z}\right)\right] \int_{-\infty}^{z} d z^{\prime}\left(z-z^{\prime}\right) \hat{\eta}_{j}\left(z^{\prime}\right) e^{i\left(M_{j}-k_{z}-\Lambda_{j z}\right)\left(z-z^{\prime}\right)}\right\} .
\end{aligned}
$$

\section{Coherence Function}

The coherence properties of signals measured at different locations and the same frequency or different frequencies and the same location give information about the ionosphere. Now that we have solutions for the electric field and the ray path, we may calculate the coherence functions.

The spatial coherence function is

$$
\Gamma_{x}\left(\vec{x}, \vec{x}^{\prime}\right)=\frac{\vec{E}(\vec{x}, \vec{k}) \cdot \vec{E}^{*}\left(\vec{x}^{\prime}, \vec{k}^{\prime}\right)}{|E(\vec{x}, \vec{k})|\left|E\left(\vec{x}^{\prime}, \vec{k}^{\prime}\right)\right|}
$$

where $\vec{k}$ is the wave vector necessary to arrive at point $\vec{x}$ and $\vec{k}^{\prime}$ is the wave vector necessary to arrive at $\vec{x}^{\prime}$. In most cases the points will be close enough together that the two $k$ vectors will be nearly identical. Omitting the polarization mixing terms, we see that

$$
\Gamma_{x}\left(\vec{x}, \vec{x}^{\prime}\right)=e^{i\left(\vec{k} \cdot \vec{x}-\vec{k}^{\prime} \cdot \vec{x}^{\prime}\right)} e^{\operatorname{Im}\left[a(\vec{x}, \vec{k})+a^{*}\left(\vec{x}^{\prime}, \vec{k}^{\prime}\right)\right]} .
$$

The value of $\Gamma_{x}$ depends on the orientation of the line between the points $\vec{x}$ and $\vec{x}^{\prime}$ with respect to the ray path. This dependence can be removed if we require this line and the ray path to be orthogonal. Assuming that $\vec{k}=\vec{k}^{\prime}$ and choosing $\vec{x}^{\prime}$ such that $\vec{k} \cdot\left(\vec{x}-\vec{x}^{\prime}\right)=0$, we have that

$$
\Gamma_{x}\left(\vec{x}, \vec{x}^{\prime}\right)=e^{\operatorname{Im}\left[a(\vec{x}, \vec{k})+a^{*}\left(\vec{x}^{\prime}, \vec{k}\right)\right]} .
$$

The distance $\left|\vec{x}^{\prime}-\vec{x}\right|$ at which the real part of this function drops to a value of $1 / e$ is called the coherence length of the ionosphere.

The frequency-coherence function is

$$
\begin{aligned}
\Gamma_{f}\left(f_{1}, f_{2}\right) & =\frac{\vec{E}\left(\vec{x}, \vec{k}_{1}^{\prime}\right) \cdot \vec{E}^{*}\left(\vec{x}, \vec{k}_{2}^{\prime}\right)}{\left|E\left(\vec{x}, \vec{k}_{1}^{\prime}\right) \| E\left(\vec{x}, \vec{k}_{2}^{\prime}\right)\right|} \\
& \approx e^{i \vec{x} \cdot\left(\vec{k}_{1}-\vec{k}_{2}\right)} e^{\operatorname{Im}\left[a\left(\vec{x}_{,}, \vec{k}_{1}\right)+a^{*}\left(\vec{x}, \vec{k}_{2}\right)\right]} e^{i \vec{x} \cdot\left(\delta \vec{k}_{1}-\delta \vec{k}_{2}\right)} e^{\operatorname{Im}\left[\vec{\nabla}_{k} a\left(\vec{x}, \vec{k}_{1}\right) \cdot \delta \vec{k}_{1}+\vec{\nabla}_{k} a^{*}\left(\vec{x}, \vec{k}_{2}\right) \cdot \delta \vec{k}_{2}\right]},
\end{aligned}
$$

where $\vec{k}_{1}^{\prime}$ and $\vec{k}_{2}^{\prime}$ are the wave vectors required for the true ray paths to arrive at $\vec{x}$ and $\vec{k}_{1}$ and $\vec{k}_{2}$ are the corresponding straight-line wave vectors. The first term in the expression for $\Gamma_{f}$ is present 
even in the absence of an ionosphere and amounts to a constant time delay of the signal in the time domain. The remaining terms are true ionospheric effects; the second term arises from dispersion, and the third and fourth terms are caused by refraction. The frequency-coherence bandwidth is defined as the difference between the frequencies $f_{1}$ and $f_{2}$ at which $\operatorname{Re}(\Gamma)$ drops to $1 / e$. This bandwidth can be enlarged if the dispersion and refraction effects are known and compensations for them can be can made. As an example, the first-order dispersion correction made for the global positioning satellites attempts to remove the $\operatorname{Im}\left[a_{1}\left(\vec{x}, \vec{k}_{1}\right)+a_{1}^{*}\left(\vec{x}, \vec{k}_{2}\right)\right]$ contribution to the phase.

\section{Laminar Ionosphere Without Waves}

In the previous sections we developed a general solution for an electric field propagating in a laminar ionosphere with waves. Although the procedure for calculating the field is straightforward, the expressions quickly become quite complicated, and it is difficult to see the physical interpretation of various terms. In this section we show how our results simplify for the case where there are no waves, and we can more easily understand the origins of the terms in the expansion.

The expression for $a_{1}$ in a laminar ionosphere with waves is given by Eq. 37, which we repeat here:

$$
a_{1}(\vec{x})=\frac{i k^{2}}{2} \sum_{j} \frac{e^{i \vec{x} \cdot \vec{\Lambda}_{j}}}{M_{j}} \int_{-\infty}^{z} d z^{\prime} \eta_{j}\left(z^{\prime}\right) e^{i\left(M_{j}-k_{z}-\Lambda_{j z}\right)\left(z-z^{\prime}\right)}
$$

A purely laminar ionosphere is obtained by setting $\vec{\Lambda}_{j}=0$, which implies that $M_{j}=k_{z}$. We then have that

$$
a_{1}(\vec{x})=\frac{i k^{2}}{2 k_{z}} \int_{-\infty}^{z} d z^{\prime} \eta\left(z^{\prime}\right)
$$

Notice that $a_{1}$ is pure imaginary, so that the effect of the ionosphere is to change only the phase of the field. The phase change is proportional to the line integral of the dielectric function along the line of sight, a well-known result.

The gradient of $a_{1}$ is

$$
\vec{\nabla} a_{1}=\frac{i k^{2} \eta(z)}{2 k_{z}} \hat{z} .
$$

Inserting this in the expression for $a_{2}$, we see that

$$
a_{2}(\vec{x})=\frac{-i k^{4}}{8 k_{z}^{3}} \int_{-\infty}^{z} d z^{\prime} \eta^{2}\left(z^{\prime}\right)
$$

Finally, the expression for $\vec{A}_{1}^{(1)}$ is

$$
\vec{A}_{1}^{(1)}(\vec{x})=\frac{\vec{E}_{0} \eta(z) e^{a_{1}(z)}}{2}
$$


The first-order equations for the horizontal displacement simplify to

$$
\begin{aligned}
\frac{d X}{d z} & =\frac{k_{x}}{k_{z}}-\frac{\eta k_{x} k^{2}}{2 k_{z}^{3}} \\
\frac{d Y}{d z} & =\frac{k_{y}}{k_{z}}-\frac{\eta k_{y} k^{2}}{2 k_{z}^{3}}
\end{aligned}
$$

These equations may easily be integrated to give the displacements. We assume that the propagating field originates at a point $\vec{x}_{0}$ located below the bottom edge of the ionosphere. We have that

$$
\begin{aligned}
& X\left(z_{o b s}\right)=x_{0}+\frac{k_{x}}{k_{z}}\left(z_{o b s}-z_{0}\right)-\frac{k^{2} k_{x}}{2 k_{z}^{3}} \int_{z_{0}}^{z} \eta\left(z^{\prime}\right) d z^{\prime} \\
& Y\left(z_{o b s}\right)=y_{0}+\frac{k_{y}}{k_{z}}\left(z_{o b s}-z_{0}\right)-\frac{k^{2} k_{y}}{2 k_{z}^{3}} \int_{z_{0}}^{z} \eta\left(z^{\prime}\right) d z^{\prime} .
\end{aligned}
$$

In order to simplify the notation, we define $T_{1}(z)=\int_{z_{0}}^{z} \eta\left(z^{\prime}\right) d z^{\prime}$ and $T_{2}(z)=\int_{z_{0}}^{z} \eta^{2}\left(z^{\prime}\right) d z^{\prime}$. The deviation of the ray path from a straight line is

$$
\vec{\Delta}_{r}(z)=\frac{-k^{2} T_{1}(z)}{2 k_{z}^{3}\left(z-z_{0}\right)}\left(k_{x}, k_{y}, 0\right) .
$$

From this we can find the change in the wave vector required to arrive at the correct endpoint, and we see that

$$
\delta \vec{k}(z)=\frac{T_{1}(z)}{2\left(z-z_{0}\right)}\left[k_{x}, k_{y}, \frac{-\left(k_{x}^{2}+k_{y}^{2}\right)}{k_{z}}\right] .
$$

The gradient of $a_{1}$ in $k$ space is

$$
\vec{\nabla}_{k} a_{1}(z)=\frac{i}{2 k_{z}}\left[2 \vec{k} T_{1}(z)+k^{2} \vec{\nabla}_{k} T_{1}(z)-\frac{k^{2} T_{1}(z)}{k_{z}} \hat{z}\right] .
$$

Using all of these results in the expression for the accumulated phase of the field, we find that

$$
\begin{aligned}
\Phi(\vec{x})= & \vec{k} \cdot\left(\vec{x}-\vec{x}_{0}\right)+\frac{k^{2} T_{1}(z)}{2 k_{z}}-\frac{k^{4} T_{2}(z)}{8 k_{z}^{3}}+\frac{k^{2} T_{1}(z)}{4 k_{z}\left(z-z_{0}\right)}\left[\vec{k} \cdot \vec{\nabla}_{k} T_{1}(z)-\frac{k^{2}}{k_{z}} \frac{\partial T_{1}(z)}{\partial k_{z}}\right] \\
& +\frac{k^{2}\left(k^{2}-k_{z}^{2}\right) T_{1}^{2}(z)}{4 k_{z}^{3}\left(z-z_{0}\right)} .
\end{aligned}
$$

It is instructive to look at the origin of each of the terms in this expression. The first term is the vacuum phase change caused by the fact that the wave has propagated away from the source 
point. The second term is the first-order dispersion caused by the nonzero index of refraction of the ionosphere and is proportional to the line integral of the dielectric function. The third term is a second-order dispersion effect and is proportional to the line integral of the square of the dielectric function. The fourth and fifth terms are caused by refraction and depend on the square of the integral of the dielectric function. The fourth term is zero if the dielectric function depends only on the magnitude of $\vec{k}$, as is usually the case in optics. The ionosphere is birefringent, however: the dielectric function is not isotropic in $k$ space, and so this term must be retained. The fifth term accounts for the refraction that occurs even in an isotropic medium. We note that this term is zero if the ray is incident normally on the ionosphere $\left(k=k_{z}\right)$. In acldition we note that the refractive terms are the only components of the phase which depend on $z$ in a way other than through limits of integration. Once the ray has passed through the ionosphere, the integrals reach limiting values and do not change further as the field continues to propagate, while the refractive terms continue to decrease as $z$ increases. Thus we see that refractive effects are most important if the observation point is close to the boundary of the ionosphere and become relatively smaller as the observation point moves farther away, unlike the pure dispersion contributions to the phase.

\section{Expansion in Inverse Powers of Frequency}

The dispersive effects of the ionosphere are frequently described in terms of an expansion in inverse powers of the wave frequency $f$. The formalism developed above is valid for any dielectric function with the postulated properties $(\eta=0$ at $z= \pm \infty, \eta \ll 1.0, \eta \rightarrow 0$ as $f \rightarrow \infty)$. The explicit coefficients in our expansion are all first order in frequency, but there is a further dependence on $f$ through the dielectric function. In order to calculate the expansion in powers of frequency, we must have the functional form of the dielectric function. In this section we will use the Appleton-Hartree expression for a collisionless ionosphere (Ref. 3):

$$
\eta(\omega)=\frac{-X(1-X)}{1-X-0.5 Y^{2} \sin ^{2} \Theta \pm \sqrt{0.25 Y^{4} \sin ^{4} \Theta+Y^{2} \cos ^{2} \Theta(1-X)^{2}}}
$$

where $X=\omega_{p}^{2} / \omega^{2}, Y=e B / m \omega$, and $\Theta$ is the angle between the ray path and the local magnetic field direction. Here $e$ and $m$ are the magnitudes of the electron charge and mass, $B$ is the magnetic induction of the local magnetic field, $\omega$ is the circular frequency of the propagating field, and $\omega_{p}$ is the local plasma frequency. The plasma frequency is $\omega_{p}^{2}=n_{e}(\vec{x}) e^{2} / \varepsilon_{0} m$, where $n_{e}$ is the local free electron density and $\varepsilon_{0}$ is the permittivity of free space. The choice of signs for the square-root term reflects the fact that the medium is birefringent and has different dielectric properties for right- and left-hand polarized waves. We shall assume that $\omega \gg e B / m$ and that the propagation direction is not nearly parallel to $\vec{B}$, so that the second term in the square root is large compared 
to the first term. Expanding the above expression to fourth order in $1 / \omega$, we have

$$
\eta(\omega) \approx-\frac{\omega_{p}^{2}}{\omega^{2}} \pm \frac{\omega_{p}^{2} e B \cos \Theta}{m \omega^{2}}-\frac{\omega_{p}^{2} e^{2} B^{2}}{m^{2} \omega^{4}}\left(1+\frac{\cos ^{2} \Theta}{2}\right) .
$$

We note that the second and third terms would be zero in the absence of a magnetic field.

We now insert the expression for $\eta$ into the formula for the phase $\Phi$ in order to get the desired expansion in inverse powers of frequency. Although this is possible for the full expression, the resulting expansion is quite messy. It will suffice for our purposes to use the simpler expressions for the case of a laminar ionosphere. We define $\mu=k_{z} / k$. Using the fact that $k=2 \pi f / c$, inserting the expression for $\eta$ into Eq. 85, and keeping terms up to order $1 / f^{3}$, we obtain

$$
\begin{aligned}
\Phi(\vec{x}) \approx & \frac{2 \pi\left(z-z_{0}\right) f}{\mu c} \\
& -\frac{e^{2} \int n_{e}\left(z^{\prime}\right) d z^{\prime}}{4 \pi \varepsilon_{0} \mu m c f} \\
& \pm \frac{e^{3} \int B\left(z^{\prime}\right) n_{e}\left(z^{\prime}\right) \cos \Theta\left(z^{\prime}\right) d z^{\prime}}{8 \pi^{2} \varepsilon_{0} \mu m^{2} c f^{2}} \\
& -\frac{e^{4} \int B^{2}\left(z^{\prime}\right) n_{e}\left(z^{\prime}\right)\left(1+0.5 \cos ^{2} \Theta\left(z^{\prime}\right)\right) d z^{\prime}}{16 \pi^{3} \varepsilon_{0} \mu m^{3} c f^{3}} \\
& -\frac{e^{4} \int n_{e}^{2}\left(z^{\prime}\right) d z^{\prime}}{64 \pi^{3} \varepsilon_{0}^{2} \mu^{3} m^{2} c f^{3}}+\frac{e^{4}\left(1-\mu^{2}\right)\left(\int n_{e}\left(z^{\prime}\right) d z^{\prime}\right)^{2}}{32 \pi^{3} \varepsilon_{0}^{2} \mu^{3} m^{2} c f^{3}\left(z-z_{0}\right)}
\end{aligned}
$$

The expansion in powers of frequency mixes orders of the previous expansion. The third-order terms on the fourth line of the above expression are generated by the expansion for $\eta$ and are part of the first-order term in the general solution. The terms on the fifth line involve only the first term in the expansion for $\eta$ but come from the higher-order terms in the general solution. We see that magnetic field effects begin to play a role at second order, while at third order there are contributions from magnetic field effects, second-order dispersion, and refraction.

It is also frequently of interest to calculate the time $\tau$ it takes for the field to propagate from the source to the observation point. This is given by the derivative of the phase with respect to angular frequency:

$$
\begin{aligned}
\tau(\vec{x}, f)=\frac{1}{2 \pi} \frac{\partial \Phi}{\partial f} \approx & \frac{z-z_{0}}{\mu c} \\
& +\frac{e^{2} \int n_{e}\left(z^{\prime}\right) d z^{\prime}}{8 \pi^{2} \varepsilon_{0} \mu m c f^{2}} \\
& \mp \frac{e^{3} \int B\left(z^{\prime}\right) n_{e}\left(z^{\prime}\right) \cos \Theta\left(z^{\prime}\right) d z^{\prime}}{8 \pi^{3} \varepsilon_{0} \mu m^{2} c f^{3}}
\end{aligned}
$$




$$
\begin{aligned}
& +\frac{3 e^{4} \int B^{2}\left(z^{\prime}\right) n_{e}\left(z^{\prime}\right)\left(1+0.5 \cos ^{2} \Theta\left(z^{\prime}\right)\right) d z^{\prime}}{32 \pi^{4} \varepsilon_{0} \mu m^{3} c f^{4}} \\
& +\frac{3 e^{4} \int n_{e}^{2}\left(z^{\prime}\right) d z^{\prime}}{128 \pi^{4} \varepsilon_{0}^{2} \mu^{3} m^{2} c f^{4}}-\frac{3 e^{4}\left(1-\mu^{2}\right)\left(\int n_{e}\left(z^{\prime}\right) d z^{\prime}\right)^{2}}{64 \pi^{4} \varepsilon_{0}^{2} \mu^{3} m^{2} c f^{4}\left(z-z_{0}\right)} .
\end{aligned}
$$

Let us plug in some typical numbers to get a feeling for the relative sizes of the various terms. The constants in SI units are $c=2.998 \times 10^{8} \mathrm{~m} / \mathrm{s}, e=1.6 \times 10^{-19} \mathrm{C}, m=9.11 \times 10^{-31} \mathrm{~kg}$, and $\varepsilon_{0}=8.85 \times 10^{-12} \mathrm{C}^{2} \mathrm{~s}^{2} / \mathrm{kg}-\mathrm{m}^{3}$. We will take the frequency of the field to be $50 \mathrm{MHz}$ and consider an observing altitude $z-z_{0}$ of $1000 \mathrm{~km}$, with $\mu=0.87$. We use an intermediate value for the magnetic induction of $3.0 \times 10^{-5} \mathrm{~T}$, and assume that the field is uniform in direction and magnitude over the volume of interest, with $\cos \theta=0.87$. The integral of the electron density is a frequently measured quantity, usually referred to as the total electron content (TEC), and we will use an intermediate TEC value of $10^{17} \mathrm{~m}^{-2}$. The integral of the square of the electron density is not an experimentally known quantity; we will estimate it by assuming we have a uniform slab $100 \mathrm{~km}$ thick with an electron density of $10^{12} \mathrm{~m}^{-3}$, giving a value of $10^{29} \mathrm{~m}^{-5}$. Plugging these values into our expression for the phase, we find that

$$
\Phi=1.21 \times 10^{6}-1.94 \times 10^{3} \pm 28.4-0.75-20.5+1.03 \mathrm{rad},
$$

where the contributions from each term are in the same order as in the preceding equation. We see that the second- and third-order contributions to the phase are indeed small compared to the first-order term but still amount to several cycles, enough to affect the coherence of the signal. The arrival time in this case is

$$
\tau=3.83 \times 10^{-3}+6.17 \times 10^{-6} \mp 1.80 \times 10^{-7}+7.16 \times 10^{-9}+1.97 \times 10^{-7}-9.92 \times 10^{-9} \mathrm{~s} .
$$

\section{Example: Parabolic Layer}

As an example we consider the case of a notional ionosphere consisting of a single parabolic layer with no waves. The electron density in this simple model depends only on altitude and is

$$
\begin{aligned}
& z_{0} \leq z<z_{1}: n_{e}(\vec{x})=0 \\
& z_{1} \leq z \leq z_{2}: n_{e}(\vec{x})=\frac{4 n_{0}\left(z-z_{1}\right)\left(z_{2}-z\right)}{\left(z_{2}-z_{1}\right)^{2}} \\
& z_{2}<z \leq z_{3}: n_{e}(\vec{x})=0 .
\end{aligned}
$$

Here $z_{0}$ is the altitude at which the signal originates, $z_{1}$ is the bottom of the electron layer, $z_{2}$ is the top of the electron layer, and $z_{3}$ is the observation altitude. We choose representative values of $z_{0}=0 \mathrm{~km}, z_{1}=250 \mathrm{~km}, z_{2}=350 \mathrm{~km}, z_{3}=800 \mathrm{~km}$, and $n_{0}=1.5 \times 10^{12} \mathrm{e} / \mathrm{m}^{3}$, giving a TEC of 
$1.0 \times 10^{17} \mathrm{e} / \mathrm{m}^{2}$. We use the Appleton-Hartree expression for the dielectric function introduced in the last section. In the absence of a magnetic field, we have that

$$
\eta(\vec{x})=-\frac{n_{e}(\vec{x}) e^{2}}{4 \pi^{2} \varepsilon_{0} m f^{2}} .
$$

With this choice of parameters, the plasma frequency at the peak of the electron layer is about 11 $\mathrm{MHz}$, and the peak value of the dielectric function is 0.048 at $50 \mathrm{MHz}$. Defining $\eta_{0}=-n_{0} e^{2} / 4 \pi^{2} \varepsilon_{0} m f^{2}$ we have that

$$
\begin{aligned}
z_{0} \leq z<z_{1}: \eta(\vec{x}) & =0 \\
z_{1} \leq z \leq z_{2}: \eta(\vec{x}) & =\frac{4 \eta_{0}\left(z-z_{1}\right)\left(z_{2}-z\right)}{\left(z_{2}-z_{1}\right)^{2}} \\
z_{2}<z \leq z_{3}: \eta(\vec{x}) & =0
\end{aligned}
$$

Inserting this in the expression for $a_{1}$, we find that

$$
\begin{aligned}
& z_{0} \leq z<z_{1}: a_{1}(z)=0 \\
& z_{1} \leq z \leq z_{2}: a_{1}(z)=\frac{i k^{2} \eta_{0}\left(z-z_{1}\right)^{2}\left(3 z_{2}-2 z-z_{1}\right)}{3 k_{z}\left(z_{2}-z_{1}\right)^{2}} \\
& z_{2}<z \leq z_{3}: a_{1}(z)=\frac{i k^{2} \eta_{0}\left(z_{2}-z_{1}\right)}{3 k_{z}}
\end{aligned}
$$

Calculating $a_{2}$ we obtain

$$
\begin{aligned}
& z_{0} \leq z<z_{1}: a_{2}(z)=0 \\
& z_{1} \leq z \leq z_{2}: a_{2}(z)=\frac{-i k^{4} \eta_{0}^{2}\left(z-z_{1}\right)^{3}\left(10 z_{2}^{2}+z_{1}^{2}+6 z^{2}+3 z z_{1}-15 z z_{2}-5 z_{1} z_{2}\right)}{15 k_{z}^{3}\left(z_{2}-z_{1}\right)^{4}} \\
& z_{2}<z \leq z_{3}: a_{2}(z)=\frac{-i k^{4} \eta_{0}^{2}\left(z_{2}-z_{1}\right)}{15 k_{z}^{3}}
\end{aligned}
$$

Solving the equation for $\overrightarrow{A_{1}}$ yields

$$
\begin{aligned}
& z_{0} \leq z<z_{1}: \vec{A}_{1}(\vec{x})=0 \\
& z_{1} \leq z \leq z_{2}: \vec{A}_{1}(\vec{x})=\frac{2 \eta_{0}\left(z-z_{1}\right)\left(z_{2}-z\right) e^{a_{1}(z)}}{\left(z_{2}-z_{1}\right)^{2}} \\
& z_{2}<z \leq z_{3}: \vec{A}_{1}(\vec{x})=0
\end{aligned}
$$

Let us now turn to the ray paths. Plugging the form for $\eta$ into the expressions for the ray path, we find

$$
z_{0} \leq z<z_{1}: X(z)=x_{0}+\frac{k_{x}\left(z-z_{0}\right)}{k_{z}}
$$




$$
\begin{aligned}
& z_{1} \leq z \leq z_{2}: X(z)=x_{0}+\frac{k_{x}\left(z-z_{0}\right)}{k_{z}}-\frac{k_{x} k^{2} \eta_{0}\left(z-z_{1}\right)^{2}\left(3 z_{2}-2 z-z_{1}\right)}{3 k_{z}^{3}\left(z_{2}-z_{1}\right)^{2}} \\
& z_{2}<z \leq z_{3}: X(z)=x_{0}+\frac{k_{x}\left(z-z_{0}\right)}{k_{z}}-\frac{k_{x} k^{2} \eta_{0}\left(z_{2}-z_{1}\right)}{3 k_{z}^{3}} \\
& z_{0} \leq z<z_{1}: Y(z)=y_{0}+\frac{k_{y}\left(z-z_{0}\right)}{k_{z}} \\
& z_{1} \leq z \leq z_{2}: Y(z)=y_{0}+\frac{k_{y}\left(z-z_{0}\right)}{k_{z}}-\frac{k_{y} k^{2} \eta_{0}\left(z-z_{1}\right)^{2}\left(3 z_{2}-2 z-z_{1}\right)}{3 k_{z}^{3}\left(z_{2}-z_{1}\right)^{2}}, \\
& z_{2}<z \leq z_{3}: Y(z)=y_{0}+\frac{k_{y}\left(z-z_{0}\right)}{k_{z}}-\frac{k_{y} k^{2} \eta_{0}\left(z_{2}-z_{1}\right)}{3 k_{z}^{3}}
\end{aligned}
$$

We see that the ray path deviates from a straight line while in the ionosphere and then emerges parallel to its original direction but displaced laterally: it has been refracted. Assuming a propagation path inclined at $30^{\circ}$ from vertical and using the ionospheric parameters given above, we find a lateral displacement of $1.23 \mathrm{~km}$. Calculating the altitude-scaled displacement $\vec{\Delta}_{r}$, we have that

$$
\vec{\Delta}_{r}=\frac{-k^{2} \eta_{0}\left(z_{2}-z_{1}\right)}{3 k_{z}^{2}\left(z_{3}-z_{0}\right)}\left(k_{x}, k_{y}, 0\right)
$$

We can calculate the change in the original wave vector required to have the endpoint of the ray path at $z=z_{3}$ be the same as the original ray path would have been without refraction:

$$
\delta \vec{k}=\frac{\eta_{0}\left(z_{2}-z_{1}\right)}{3\left(z_{3}-z_{0}\right)}\left(k_{x}, k_{y}, \frac{-\left(k_{x}^{2}+k_{y}^{2}\right)}{k_{z}}\right) .
$$

Replacing $\vec{k}$ with $\vec{k}+\delta \vec{k}$ in the expressions for the ray path, we find the corrected ray paths:

$$
\begin{aligned}
z_{0} \leq z<z_{1}: X(z) & =x_{0}+\frac{k_{x}\left(z-z_{0}\right)}{k_{z}}+\frac{k_{x} k^{2} \eta_{0}\left(z_{2}-z_{1}\right)\left(z-z_{0}\right)}{3 k_{z}^{3}\left(z_{3}-z_{0}\right)} \\
z_{1} \leq z \leq z_{2}: X(z)= & x_{0}+\frac{k_{x}\left(z-z_{0}\right)}{k_{z}}+\frac{k_{x} k^{2} \eta_{0}\left(z_{2}-z_{1}\right)\left(z-z_{0}\right)}{3 k_{z}^{3}\left(z_{3}-z_{0}\right)} \\
& -\frac{k_{x} k^{2} \eta_{0}\left(z-z_{1}\right)^{2}\left(3 z_{2}-2 z-z_{1}\right)}{3 k_{z}^{3}\left(z_{2}-z_{1}\right)^{2}} \\
z_{2}<z \leq z_{3}: X(z)= & x_{0}+\frac{k_{x}\left(z-z_{0}\right)}{k_{z}}+\frac{k_{x} k^{2} \eta_{0}\left(z_{2}-z_{1}\right)\left(z-z_{0}\right)}{3 k_{z}^{3}\left(z_{3}-z_{0}\right)}-\frac{k_{x} k^{2} \eta_{0}\left(z_{2}-z_{1}\right)}{3 k_{z}^{3}} \\
z_{0} \leq z<z_{1}: Y(z)= & y_{0}+\frac{k_{y}\left(z-z_{0}\right)}{k_{z}}+\frac{k_{y} k^{2} \eta_{0}\left(z_{2}-z_{1}\right)\left(z-z_{0}\right)}{3 k_{z}^{3}\left(z_{3}-z_{0}\right)}
\end{aligned}
$$




$$
\begin{aligned}
z_{1} \leq z \leq z_{2}: Y(z) & =y_{0}+\frac{k_{y}\left(z-z_{0}\right)}{k_{z}}+\frac{k_{y} k^{2} \eta_{0}\left(z_{2}-z_{1}\right)\left(z-z_{0}\right)}{3 k_{z}^{3}\left(z_{3}-z_{0}\right)} \\
& -\frac{k_{y} k^{2} \eta_{0}\left(z-z_{1}\right)^{2}\left(3 z_{2}-2 z-z_{1}\right)}{3 k_{z}^{3}\left(z_{2}-z_{1}\right)^{2}} \\
z_{2}<z \leq z_{3}: Y(z) & =y_{0}+\frac{k_{y}\left(z-z_{0}\right)}{k_{z}}+\frac{k_{y} k^{2} \eta_{0}\left(z_{2}-z_{1}\right)\left(z-z_{0}\right)}{3 k_{z}^{3}\left(z_{3}-z_{0}\right)}-\frac{k_{y} k^{2} \eta_{0}\left(z_{2}-z_{1}\right)}{3 k_{z}^{3}}
\end{aligned}
$$

Figure 1 shows the deviation of the ray path from a straight line for the model ionosphere parameters given above with the straight-line propagation path at $30^{\circ}$ from vertical, the source at $z=0$ and the observation point at $z=800 \mathrm{~km}$.

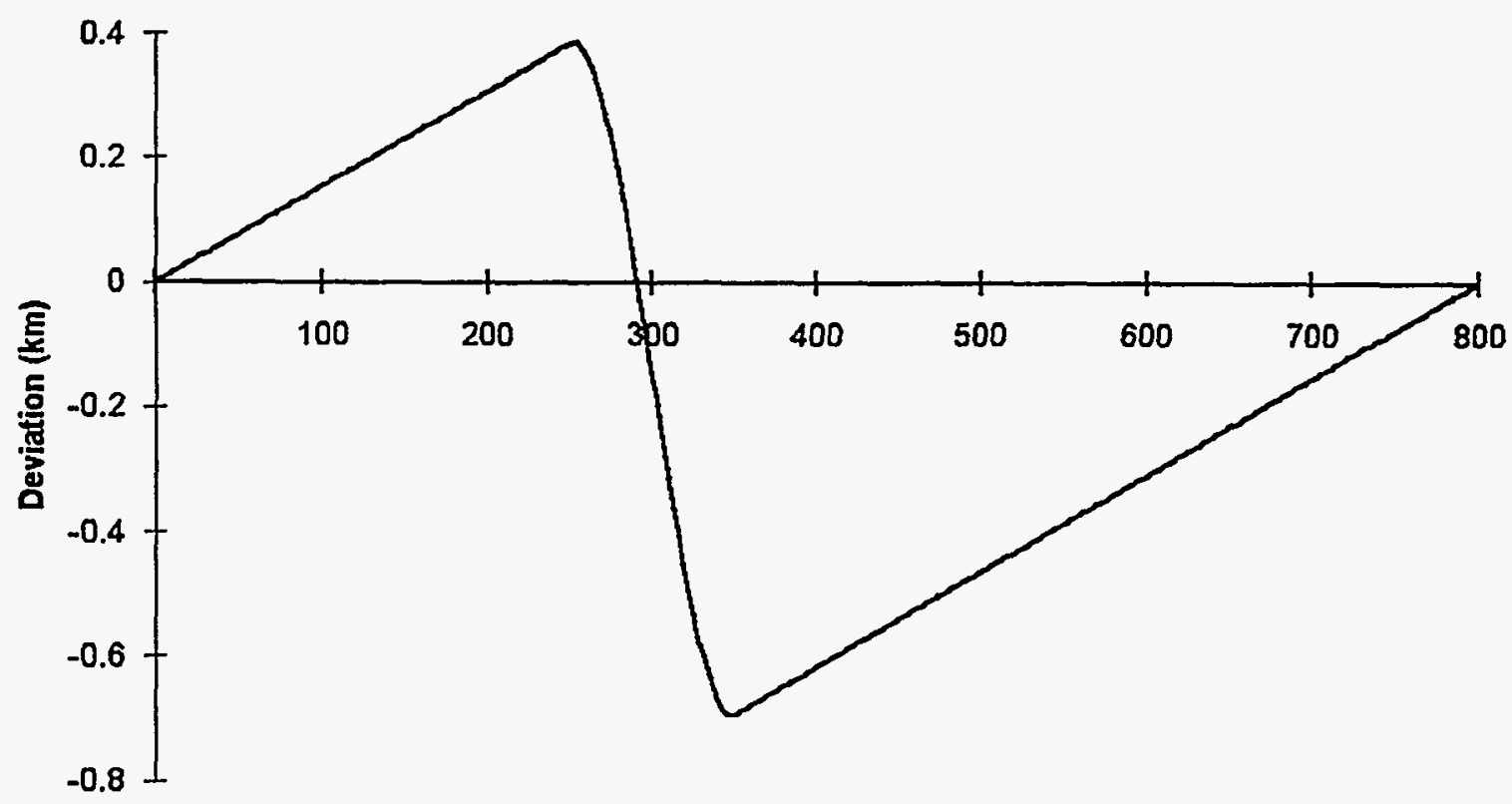

Altitude (km)

Figure 1. Deviation from straight-line path for parabolic layer model.

In order to calculate the phase of the field, we need the gradient of $a_{1}$ in $k$ space:

$$
\vec{\nabla}_{k} a_{1}=\frac{i \eta 0 k^{2}\left(z_{2}-z_{1}\right)}{3 k_{z}^{2}} \hat{z}
$$


Using this and the expressions for $a_{1}, a_{2}$, and $\vec{\delta} k$ in the equation for the phase, we obtain

$$
\Phi\left(z_{3}\right)=\vec{k} \cdot\left(\vec{x}_{o b s}-\vec{x}_{0}\right)+\frac{k^{2} \eta_{0}\left(z_{2}-z_{1}\right)}{3 k_{z}}-\frac{k^{4} \eta_{0}^{2}\left(z_{2}-z_{1}\right)}{15 k_{z}^{3}}+\frac{k^{2}\left(k^{2}-k_{z}^{2}\right) \eta_{0}^{2}\left(z_{2}-z_{1}\right)^{2}}{9 k_{z}^{3}\left(z_{3}-z_{0}\right)}
$$

Plugging in the parameter values for the model, we have

$$
\Phi=9.68 \times 10^{5}+1.94 \times 10^{3}-24.8+1.29 \mathrm{rad},
$$

where the terms are in the same order as in the previous equation.

\section{References}

[1] See for example J.D. Jackson, Classical Electrodynamics, 2nd ed., (John Wiley and Sons, New York, 1975), pp. 14-15.

[2] I.S. Gradshteyn \& I.M. Ryzhik, Table of Integrals, Series, and Products, corrected and enlarged edition, (Academic Press, San Diego, 1980).

[3] See for example K.G. Budden, The Propagation of Radio Waves, (Cambridge University Press, Cambridge, 1988), pp. 74-75. 